\title{
ON THE LENGTH OF THE LONGEST INCREASING SUBSEQUENCE IN A RANDOM PERMUTATION
}

\author{
BÉla BollobÁs AND Svante Janson \\ Dedicated to Paul Erdös on his eightieth birthday.
}

\begin{abstract}
A вstract. Complementing the results claiming that the maximal length $L_{n}$ of an increasing subsequence in a random permutation of $\{1,2, \ldots, n\}$ is highly concentrated, we show that $L_{n}$ is not concentrated in a short interval: $\sup _{l} \mathrm{P}\left(l \leq L_{n} \leq l+n^{1 / 16} \log ^{-3 / 8} n\right) \rightarrow 0$ as $n \rightarrow \infty$.
\end{abstract}

\section{INTRODUCTION}

Ulam [9] proposed the study of $L_{n}$, the maximal length of an increasing subsequence of a random permutation of the set $[n]=\{1,2, \ldots, n\}$. Hammersley [4], Logan and Shepp [7], and Veršik and Kerov [10] proved that $\mathrm{E} L_{n} \sim 2 \sqrt{n}$ and

$$
L_{n} / \sqrt{n} \stackrel{\mathrm{p}}{\rightarrow} 2 \text { as } n \rightarrow \infty .
$$

Frieze [3] showed that the distribution of $L_{n}$ is sharply concentrated about its mean; his result was improved by Bollobás and Brightwell [2], who in particular proved that

$$
\operatorname{Var}\left(L_{n}\right)=O\left(n^{1 / 2}(\log n / \log \log n)^{2}\right) .
$$

(The log factors have recently been removed by Talagrand [8].) Somewhat surprisingly, it is not known that the distribution of $L_{n}$ is not much more concentrated than claimed by (1.2). In fact, it has not even been ruled out that if $w(n) \rightarrow \infty$ then $\mathrm{P}\left(\left|L_{n}-\mathrm{E} L_{n}\right|<w(n)\right) \rightarrow 0$ as $n \rightarrow \infty$. Our aim in this paper is to rule out this possibility for a fairly fast-growing function $w(n)$, and to give a lower bound for $\operatorname{Var}\left(L_{n}\right)$, complementing (1.2).

Theorem 1.

$$
\mathrm{P}\left(\left|L_{n}-\mathrm{E} L_{n}\right| \leq n^{1 / 16} \log ^{-3 / 8} n\right) \rightarrow 0 \quad \text { as } \quad n \rightarrow \infty .
$$

More generally, if $a_{n}$ and $b_{n}$ are any numbers such that

$$
\inf \mathrm{P}\left(a_{n} \leq L_{n} \leq b_{n}\right)>0, \quad \text { then } \quad\left(b_{n}-a_{n}\right) / n^{1 / 16} \log ^{-3 / 8} n \rightarrow \infty .
$$

In particular, for sufficiently large $n$,

$$
\operatorname{Var} L_{n} \geq n^{1 / 8} \log ^{-3 / 4} n .
$$

There is still a wide gap between the upper and lower bound, and there is no reason to believe that the bounds given here are the best possible. In fact, a boot-strap argument

Second author supported by the Göran Gustafsson Foundation for Research in Natural Sciences and Medicine

Typeset by $\mathcal{A M}_{\mathcal{M}}-\mathrm{T}_{\mathrm{EX}}$ 
suggests that the range of variation is at least about $n^{1 / 10}$, see Theorem 2 below, and it is quite possible that the upper bound in (1.2) is sharp up to logarithmic factors, as conjectured in [2].

It is well-known that $L_{n}$ also can be defined as the height of the random partial order defined as follows. Consider the unit square $Q=[0,1]^{2}$ with the coordinate order. Thus for $(x, y),\left(x^{\prime}, y^{\prime}\right) \in Q$ set $(x, y) \leq\left(x^{\prime}, y^{\prime}\right)$ if and only if $x \leq x^{\prime}$ and $y \leq y^{\prime}$, let $\left(\xi_{i}\right)_{i=1}^{\infty}$ be independent, uniformly distributed random points in $Q$ and consider the induced partial order on the set $\left(\xi_{i}\right)_{i=1}^{n}$.

Let $\mu>0$ be a constant and let $m$ be the Lebesque measure in $Q$. Let us regard a Poisson process with intensity $\mu d m$ in $Q$ as a random subset of $Q$. Equivalently, let $N$ be independent of $\left(\xi_{i}\right)_{1}^{\infty}$, with distribution $\operatorname{Po}(\mu)$, and take the set $\left\{\xi_{i}: 1 \leq i \leq N\right\}$. Write $H_{\mu}$ for the height of the induced partial order on this set.

In [2] the proof of (1.2) was based on a study of $H_{n}$. In particular they proved that

$$
\mathrm{P}\left(\left|H_{n}-\mathrm{E} H_{n}\right|>K_{1} \lambda \frac{n^{1 / 4} \log n}{\log \log n}\right) \leq e^{-\lambda^{2}}
$$

for some constant $K_{1}$, every $n \geq 3$ and every $\lambda$ with $1 \leq \lambda \leq n^{1 / 4} / \log \log n$. For larger $\lambda$ their proof yields

$$
\mathrm{P}\left(\left|H_{n}-\mathrm{E} H_{n}\right|>K_{2} \lambda^{2} \log \lambda\right) \leq e^{-\lambda^{2}} .
$$

These inequalities hold for non-integer $n$ as well: and that if $n \geq 3$ and $1 \leq \lambda \leq$ $n^{1 / 4} / \log \log n$, then for every $\mu \leq n$, we have

$$
\mathrm{P}\left(\left|H_{\mu}-\mathrm{E} H_{\mu}\right|>K_{3} \lambda \frac{n^{1 / 4} \log n}{\log \log n}\right) \leq e^{-\lambda^{2}}
$$

It is rather curious that our proof of a lower bound will use these results together with, as well as the following estimate from [2]:

$$
0 \leq 2 n^{1 / 2}-\mathrm{E} H_{n} \leq K_{4} n^{1 / 4} \log ^{3 / 2} n / \log \log n
$$

Remark. It is shown in [2] that (1.3) holds for $L_{n}$ as well. (The same is true for (1.4) and (1.5).) Similarly, Theorem 1 holds for $H_{n}$ too; this follows from the proof of Theorem 1 below, with a few simplifications.

The variables $L_{n}$ and $H_{n}$ may be defined, more generally, for random subsets of the $d$-dimensional cube $[0,1]^{d}$. The results in [2] include this generalization, and it would be interesting to find lower bounds for the variance. Unfortunately, and somewhat surprisingly, the method used here does not work when $d \geq 3$. We try to explain this failure at the end of the paper.

\section{Proof of THEOREM 1}

The idea behind the proof is that $L_{n}$ essentially depends only on the points in a strip of measure $n^{-\alpha}$ for some $\alpha>0(\alpha=1 / 8$ if we ignore logarithmic factors). The number of points in this strip is approximately Poisson distributed with expectation $n^{1-\alpha}$; hence the random variation of this number is of order $n^{(1-\alpha) / 2}$ and the relative variation is $n^{-(1-\alpha) / 2}$. This ought to correspond to a relative variation in the height of the same order $n^{-(1-\alpha) / 2}$, ignoring the further variation due to the random position of the points, which would give a variation of order at least $n^{1 / 2} \cdot n^{-(1-\alpha) / 2}=n^{\alpha / 2}$.

We introduce some notation. For a Borel set $S \subset Q$, let

$$
N_{n}(S)=\left|\left\{i \leq n: \xi_{i} \in S\right\}\right|
$$


be the number of those of our $n$ random points that lie in $S$, and let $L_{n}(S)$ be the height of the partial order defined by these $N_{n}(S)$ points; similarly, let $H_{n}(S)$ be the height of the partial order defined by the restriction of our Poisson process to $S$. Finally, let $S_{\delta}=\{(x, y) \in Q:|x-y| \leq \delta\}$ be the strip of width $2 \delta$ along the diagonal. We shall deduce our theorem from two lemmas. The first of these claims that the height only depends on the points in $S_{\delta}$ for a fairly small value of $\delta$.

Lemma 1. If $K$ is sufficiently large, then with $\delta_{n}=K n^{-1 / 8} \log ^{3 / 4} n(\log \log n)^{-1 / 2}$ we have

$$
\mathrm{P}\left(L_{n} \neq L_{n}\left(S_{\delta_{n}}\right)\right) \rightarrow 0 \quad \text { as } \quad n \rightarrow \infty
$$

Proof. We claim that $K=\max \left(3 K_{3}{ }^{1 / 2}, 2 K_{4}^{1 / 2}\right)$ will do, where $K_{3}$ and $K_{4}$ are the constants in (1.5) and (1.6). In fact, we shall prove slightly more than claimed, namely that the probability that the set $\left\{\xi_{i}: 1 \leq i \leq n\right\}$ contains a point $\xi_{i} \notin S_{\delta_{n}}$ that belongs to a maximal chain is $o(1)$. Since the probability that a Poisson process $\Xi$ in $Q$ with intensity $n$ has exactly $n$ points with probability at least $e^{-1} n^{-1 / 2}$, it suffices to show that the corresponding probability for the Poisson process $\Xi$ is $o\left(n^{-1 / 2}\right)$.

Let $M$ be the number of points in $\Xi \backslash S_{\delta}$ that belong to a maximal chain in $\Xi$. Then

$$
M=\sum_{\xi \in \Xi} f(\xi, \Xi)
$$

where

$$
f(\xi, \Xi)=I\left(\xi \notin S_{\delta}\right) \cdot I(\xi \text { belongs to a maximal chain in } \Xi) .
$$

Hence, using an easily proved formula for Poisson processes (see, e.g., [5, Lemma 2.1], and [6, Lemma 10.1 and Exercise 11.1]),

$$
\begin{aligned}
\mathrm{E} M & =\mathrm{E} \sum_{\xi \in \Xi} f(\xi, \Xi)=\int_{Q} \mathrm{E} f(z, \Xi \cup\{z\}) n d m(z) \\
& =\int_{Q \backslash S_{\delta}} \mathrm{P}(z \text { belongs to a maximal chain in } \Xi \cup\{z\}) n d m(z) .
\end{aligned}
$$

Fix $z=(x, y) \notin S_{\delta}$ and let $s=(x+y) / 2, t=(x-y) / 2, Q_{1}=[0, x] \times[0, y]$ and $Q_{2}=[x, 1] \times[y, 1]$. Then, writing $|R|$ for the area of a set $R \subset Q$, we have

$$
\begin{aligned}
\left|Q_{1}\right|^{1 / 2}+\left|Q_{2}\right|^{1 / 2} & =\left(s^{2}-t^{2}\right)^{1 / 2}+\left((1-s)^{2}-t^{2}\right)^{1 / 2} \\
& \leq s-\frac{t^{2}}{2 s}+1-s-\frac{t^{2}}{2(1-s)} \\
& =1-\frac{t^{2}}{2 s(1-s)} \\
& \leq 1-2 t^{2} \\
& \leq 1-\frac{1}{2} \delta^{2}
\end{aligned}
$$

The random variables $H_{n}\left(Q_{1}\right)$ and $H_{n}\left(Q_{2}\right)$ have the same distributions as $H_{\mu_{1}}$ and $H_{\mu_{2}}$, respectively, with $\mu_{i}=n\left|Q_{i}\right|, i=1,2$. Setting $\delta=\delta_{n}$, inequality (1.6) implies that if $n$ is large enough,

$$
\mathrm{E} H_{\mu_{1}}+\mathrm{E} H_{\mu_{2}} \leq 2 \mu_{1}^{1 / 2}+2 \mu_{2}^{1 / 2} \leq 2 n^{1 / 2}-n^{1 / 2} \delta_{n}^{2} \leq \mathrm{E} H_{n}-1-\frac{1}{2} n^{1 / 2} \delta_{n}^{2} .
$$


Hence, by applying (1.5) with $\lambda=(2 \log n)^{1 / 2}$, we find that

$$
\begin{aligned}
& \mathrm{P}(z \text { belongs to a maximal chain in } \Xi \cup\{z\})=\mathrm{P}\left(H_{n}\left(Q_{1}\right)+H_{n}\left(Q_{2}\right)+1 \geq H_{n}\right) \\
& \leq \mathrm{P}\left(H_{\mu_{1}} \geq \mathrm{E} H_{\mu_{1}}+\frac{1}{6} n^{1 / 2} \delta_{n}^{2}\right)+\mathrm{P}\left(H_{\mu_{2}} \geq \mathrm{E} H_{\mu_{2}}+\frac{1}{6} n^{1 / 2} \delta_{n}^{2}\right) \\
& \quad+\mathrm{P}\left(H_{n} \leq \mathrm{E} H_{n}-\frac{1}{6} n^{1 / 2} \delta_{n}^{2}\right) \\
& \leq 3 \exp (-2 \log n)=3 n^{-2} .
\end{aligned}
$$

Consequently, (2.1) yields E $M \leq 3 n^{-1}$, and the result follows.

Lemma 2. Suppose that $\delta_{n} \searrow 0$ and that $\mathrm{P}\left(L_{n} \neq L_{n}\left(S_{\delta_{n}}\right)\right) \rightarrow 0$ as $n \rightarrow \infty$. If $\left(\alpha_{n}\right)$ is any sequence with $\alpha_{n}=o\left(\delta_{n}^{-1 / 2}\right)$ then

$$
\sup _{x} \mathrm{P}\left(\left|L_{n}-x\right| \leq \alpha_{n}\right) \rightarrow 0 \quad \text { as } \quad n \rightarrow \infty
$$

Proof. It is convenient to use couplings, and we begin by recalling the relevant definitions. A coupling of two random variables $X$ and $Y$ (possibly defined on different probability spaces), is a pair of random variables $\left(X^{\prime}, Y^{\prime}\right)$ defined on a common probability space such that $X^{\prime} \stackrel{\mathrm{d}}{=} X$ and $Y^{\prime} \stackrel{\mathrm{d}}{=} Y$. The notion of coupling depends only on the distributions of $X$ and $Y$, so we may as well talk about a coupling of two distributions (which can be formulated as finding a joint distribution with given marginals).

We also define the total variation distance of two random variables $X$ and $Y$ (or, more properly, of their distributions $\mathcal{L}(X)$ and $\mathcal{L}(Y))$ as

$$
d_{T V}(X, Y)=\sup _{A}|\mathrm{P}(X \in A)-\mathrm{P}(Y \in A)|
$$

taking the supremum over all Borel sets $A$. If $\left(X^{\prime}, Y^{\prime}\right)$ is a coupling of $X$ and $Y$ then, clearly, $d_{T V}(X, Y)=d_{T V}\left(X^{\prime}, Y^{\prime}\right) \leq \mathrm{P}\left(X^{\prime} \neq Y^{\prime}\right)$. Conversely, it is easy to construct a coupling of $X$ and $Y$ such that equality holds (such couplings are known as maximal couplings). Thus

$$
d_{T V}(X, Y)=\min \mathrm{P}\left(X^{\prime} \neq Y^{\prime}\right)
$$

where the minimum ranges over all couplings of $X$ and $Y$. Moreover, provided the probability space where $X$ is defined is rich enough, there exists a maximal coupling $\left(X^{\prime}, Y^{\prime}\right)$ of $X$ and $Y$ with $X^{\prime}=X$.

We may assume that $\delta_{n}<1$ and $\alpha_{n} \geq \delta_{n}^{-1 / 4} \rightarrow \infty$. (All limits in the proof are taken as $n \rightarrow \infty$.)

Let $m=m(n)=\left\lceil 6 \alpha_{n} \sqrt{n} \mid \leq 7 \alpha_{n} \sqrt{n}\right.$, and let $\mu=\mu(n)=\left|S_{\delta_{n}}\right|$; thus

$$
\delta_{n} \leq \mu \leq 2 \delta_{n}
$$

We use the facts that, for any $n, p, \lambda_{1}, \lambda_{2}$,

$$
d_{T V}(\operatorname{Bi}(n, p), \operatorname{Po}(n p)) \leq p
$$

and

$$
d_{T V}\left(\operatorname{Po}\left(\lambda_{1}\right), \operatorname{Po}\left(\lambda_{2}\right)\right) \leq\left|\lambda_{1}-\lambda_{2}\right| / \max \left(\lambda_{1}, \lambda_{2}\right)^{1 / 2},
$$


see e.g. [1, Theorems 2.M and 1.C]. Hence

$$
\begin{aligned}
d_{T V}\left(N_{n}\left(S_{\delta_{n}}\right), N_{n+m}\left(S_{\delta_{n}}\right)\right)=d_{T V}(\operatorname{Bi}(n, \mu), \operatorname{Bi}(n+m, \mu)) \\
\leq d_{T V}(\operatorname{Bi}(n, \mu), \operatorname{Po}(n \mu))+d_{T V}(\operatorname{Po}(n \mu), \operatorname{Po}((n+m) \mu)) \\
\quad+d_{T V}(\operatorname{Po}((n+m) \mu), \operatorname{Bi}(n+m, \mu)) \\
\leq \mu+m \mu /(n \mu)^{1 / 2}+\mu \\
=m n^{-1 / 2} \mu^{1 / 2}+2 \mu \leq 7 \sqrt{2} \alpha_{n} \delta_{n}^{1 / 2}+4 \delta_{n} \leq 14 \alpha_{n} \delta_{n}^{1 / 2} .
\end{aligned}
$$

Choose a maximal coupling $\left(N_{n}^{\prime}, N_{n+m}^{\prime}\right)$ of $N_{n}\left(S_{\delta_{n}}\right)$ and $N_{n+m}\left(S_{\delta_{n}}\right)$, and let $\left(\xi_{i}^{\prime}\right)_{i=1}^{\infty}$ be a sequence of independent random points, uniformly distributed in $S_{\delta_{n}}$; assume also that $\left(\xi_{i}^{\prime}\right)$ is independent of $\left(N_{n}^{\prime}, N_{n+m}^{\prime}\right)$. Let $L^{\prime}(N)$ be the height of the partial order defined by $\left\{\xi_{i}^{\prime}: i \leq N\right\}$. Then $\left(L^{\prime}\left(N_{n}^{\prime}\right), L^{\prime}\left(N_{n+m}^{\prime}\right)\right)$ is a coupling of $L_{n}\left(S_{\delta_{n}}\right)$ and $L_{n+m}\left(S_{\delta_{n}}\right)$, and thus

$$
\begin{aligned}
d_{T V}\left(L_{n}\left(S_{\delta_{n}}\right), L_{n+m}\left(S_{\delta_{n}}\right)\right) & \leq \mathrm{P}\left(L^{\prime}\left(N_{n}^{\prime}\right) \neq L^{\prime}\left(N_{n+m}^{\prime}\right)\right) \\
& \leq \mathrm{P}\left(N_{n}^{\prime} \neq N_{n+m}^{\prime}\right)=d_{T V}\left(N_{n}\left(S_{\delta_{n}}\right), N_{n+m}\left(S_{\delta_{n}}\right)\right) \\
& \leq 14 \alpha_{n} \delta_{n}^{1 / 2} .
\end{aligned}
$$

Furthermore, using $L_{n+m}\left(S_{\delta_{n+m}}\right) \leq L_{n+m}\left(S_{\delta_{n}}\right) \leq L_{n+m}$, we see that

$$
\begin{aligned}
d_{T V} & \left(L_{n}, L_{n+m}\right) \\
& \leq \mathrm{P}\left(L_{n} \neq L_{n}\left(S_{\delta_{n}}\right)\right)+\mathrm{P}\left(L_{n+m} \neq L_{n+m}\left(S_{\delta_{n}}\right)\right)+d_{T V}\left(L_{n}\left(S_{\delta_{n}}\right), L_{n+m}\left(S_{\delta_{n}}\right)\right) \\
& \leq \mathrm{P}\left(L_{n} \neq L_{n}\left(S_{\delta_{n}}\right)\right)+\mathrm{P}\left(L_{n+m} \neq L_{n+m}\left(S_{\delta_{n+m}}\right)\right)+14 \alpha_{n} \delta_{n}^{1 / 2} \\
& \rightarrow 0 .
\end{aligned}
$$

Hence a maximal coupling $\left(L_{n}^{\prime}, L_{n+m}^{\prime}\right)$ of $L_{n}$ and $L_{n+m}$ satisfies $\mathrm{P}\left(L_{n}^{\prime} \neq L_{n+m}^{\prime}\right) \rightarrow 0$.

We next define another coupling of $L_{n}$ and $L_{n+m}$, now trying to push the variables apart. Observe that necessarily $n \delta_{n} \rightarrow \infty$, since otherwise, for some $C<\infty$ and arbitrarily large $n$,

$$
\mathrm{E} L_{n}\left(S_{\delta_{n}}\right) \leq \mathrm{E} N_{n}\left(S_{\delta_{n}}\right)=n\left|S_{\delta_{n}}\right| \leq 2 n \delta_{n} \leq 2 C,
$$

which contradicts $L_{n} / \sqrt{n} \stackrel{\mathrm{p}}{\longrightarrow} 2$ and $\mathrm{P}\left(L_{n} \neq L_{n}\left(S_{\delta_{n}}\right)\right) \rightarrow 0$. Hence $m=O\left(\alpha_{n} n^{1 / 2}\right)=$ $o\left(\delta_{n}^{-1 / 2} n^{1 / 2}\right)=o(n)$.

In particular, we may assume that $n>3 m$. Set $Q_{1}=\left[0, \frac{m}{3 n}\right]^{2}$ and $Q_{2}=\left(\frac{m}{3 n}, 1\right]^{2}$. Then

$$
L_{n+m} \geq L_{n+m}\left(Q_{1}\right)+L_{n+m}\left(Q_{2}\right) .
$$

Moreover, $N_{n+m}\left(Q_{1}\right) \sim \operatorname{Bi}\left(n+m,\left(\frac{m}{3 n}\right)^{2}\right)$ with an expectation of $(n+m)\left(\frac{m}{3 n}\right)^{2}>\frac{m^{2}}{9 n} \geq 4 \alpha_{n}^{2}$; and it follows from Chebyshev's inequality, that

$$
\mathrm{P}\left(N_{n+m}\left(Q_{1}\right) \geq 2 \alpha_{n}^{2}\right) \rightarrow 1
$$

Since the distribution of $L_{n+m}\left(Q_{1}\right)$ conditional on $N_{n+m}\left(Q_{1}\right)=\nu$ equals the distribution of $L_{\nu}$ for any $\nu \geq 1$, we obtain from (1.1) that

$$
\mathrm{P}\left(L_{n+m}\left(Q_{1}\right)>2 \alpha_{n}\right) \rightarrow 1
$$


Similarly, $n+m-N_{n+m}\left(Q_{2}\right) \sim \operatorname{Bi}\left(n+m, 1-\left(1-\frac{m}{3 n}\right)^{2}\right)$ with expectation

$$
(n+m)\left(2 \frac{m}{3 n}-\frac{m^{2}}{9 n^{2}}\right)=\left(\frac{2}{3}+o(1)\right) m
$$

and thus

$$
\mathrm{P}\left(N_{n+m}\left(Q_{2}\right) \geq n\right)=\mathrm{P}\left(n+m-N_{n+m}\left(Q_{2}\right) \leq m\right) \rightarrow 1 .
$$

We define $L_{n}^{\prime \prime}$ to be the height of the partial order defined by the first $n$ of $\xi_{1}, \xi_{2}, \ldots$ that fall in $Q_{2}$; obviously $L_{n}^{\prime \prime} \stackrel{\text { d }}{=} L_{n}$, so $\left(L_{n}^{\prime \prime}, L_{n+m}\right)$ is a coupling of $L_{n}$ and $L_{n+m}$. Moreover, if $N_{n+m}\left(Q_{2}\right) \geq n$, then $L_{n+m}\left(Q_{2}\right) \geq L_{n}^{\prime \prime}$, and thus (2.4), (2.6), (2.7) yield

$$
\mathrm{P}\left(L_{n+m}>L_{n}^{\prime \prime}+2 \alpha_{n}\right) \rightarrow 1 \text {. }
$$

Combining this coupling with a maximal coupling $\left(L_{n+m}^{\prime}, L_{n}^{\prime}\right)$ of $L_{n+m}$ and $L_{n}$ such that $L_{n+m}^{\prime}=L_{n+m}$, we obtain a coupling $\left(L_{n}^{\prime}, L_{n}^{\prime \prime}\right)$ of $L_{n}$ with itself, i.e. two random variables $L_{n}^{\prime}$ and $L_{n}^{\prime \prime}$ with $L_{n}^{\prime} \stackrel{\mathrm{d}}{=} L_{n}^{\prime \prime} \stackrel{\mathrm{d}}{=} L_{n}$, such that

$$
\mathrm{P}\left(L_{n}^{\prime}>L_{n}^{\prime \prime}+2 \alpha_{n}\right) \geq \mathrm{P}\left(L_{n+m}>L_{n}^{\prime \prime}+2 \alpha_{n}\right)-\mathrm{P}\left(L_{n+m} \neq L_{n}^{\prime}\right) \rightarrow 1 .
$$

Finally we observe that for any real $x$,

$$
\mathrm{P}\left(L_{n}^{\prime}>L_{n}^{\prime \prime}+2 \alpha_{n}\right) \leq \mathrm{P}\left(L_{n}^{\prime}>x+\alpha_{n}\right)+\mathrm{P}\left(L_{n}^{\prime \prime}<x-\alpha_{n}\right)=\mathrm{P}\left(\left|L_{n}-x\right|>\alpha_{n}\right)
$$

and thus

$$
\sup _{x} \mathrm{P}\left(\left|L_{n}-x\right| \leq \alpha_{n}\right) \leq 1-\mathrm{P}\left(L_{n}^{\prime}>L_{n}^{\prime \prime}+\alpha_{n}\right) \rightarrow 0 .
$$

Theorem 1 follows immediately from the lemmas.

\section{FURTHER REMARKS}

Note that the proof of Theorem 1 uses the concentration results in [2], and that stronger concentration results would imply a stronger version of Theorem 1, i.e. less concentration than given above. This leads to the following result, which shows that, at least for some $n$, the distribution of $H_{n}$ is not strictly concentrated (with, say, exponentially decreasing tails) with a variation of much less than $n^{-1 / 10}$. (For simplicity we consider here $H_{n}$; presumably the same result is true for $L_{n}$.)

Theorem 2. If $\varepsilon>0$ is sufficiently small, then there exist infinitely many $n$ such that for some $m \leq n$ we have

$$
\mathrm{P}\left(\left|H_{m}-\mathrm{E} H_{m}\right|>\varepsilon n^{1 / 10}\right)>n^{-2} .
$$

Proof. Assume on the contrary, and somewhat more generally, that for some $\gamma, 0<\gamma<$ $1 / 2$, and all large $n$,

$$
\mathrm{P}\left(\left|H_{m}-\mathrm{E} H_{m}\right|>n^{\gamma}\right) \leq n^{-2}, \quad m \leq n .
$$

The argument in the proof of [2, Theorem 9] then yields

$$
2 n^{1 / 2}-\mathrm{E} H_{n}=O\left(n^{\gamma}\right)
$$


and Lemma 1 holds for $H_{n}$, by the argument above, with

$$
\delta_{n}=K n^{\gamma / 2-1 / 4},
$$

provided $K$ is large enough. Hence Lemma $2\left(\right.$ for $H_{n}$ ) shows that

$$
\mathrm{P}\left(\left|H_{n}-\mathrm{E} H_{n}\right| \leq \alpha_{n}\right) \rightarrow 0
$$

whenever $\alpha_{n}=o\left(\delta_{n}^{-1 / 2}\right)$, i.e., when

$$
\alpha_{n} n^{\gamma / 4-1 / 8} \rightarrow 0
$$

If $\gamma<1 / 10$, we may take $\alpha_{n}=n^{\gamma}$, which then satisfies (3.5), and obtain a contradiction from (3.1) and (3.4). In order to obtain the slightly stronger statement in the theorem, we let $\gamma=1 / 10$ and note that if

$$
\mathrm{P}\left(\left|H_{n}-\mathrm{E} H_{n}\right|>\varepsilon n^{1 / 10}\right) \leq n^{-2}<1 / 2
$$

for every $\varepsilon>0$ and $n \geq n(\varepsilon)$, then there exists a sequence $\varepsilon_{n} \rightarrow 0$ such that

$$
\mathrm{P}\left(\left|H_{n}-\mathrm{E} H_{n}\right|>\varepsilon_{n} n^{1 / 10}\right)<1 / 2 .
$$

We now choose $\alpha_{n}=\varepsilon_{n} n^{1 / 10}$, which satisfies (3.5), and obtain a contradiction from (3.4) and (3.7). Hence either (3.1) or (3.6), for some $\varepsilon>0$, fails for infinitely many $n$, which proves the result.

Finally, let us see what happens when we try to generalize the results to the random $d$-dimensional order defined by random points in $Q_{d}=[0,1]^{d}$. Lemma 1 holds, with

$$
\delta_{n}=K n^{-1 / 4 d} \log ^{3 / 4} n(\log \log n)^{-1 / 2},
$$

by essentially the same proof; we now define $S_{\delta}=\left\{\left(x_{i}\right)^{d}:\left|x_{i}-x_{j}\right| \leq \delta, i<j\right\}$, and note that $\left|S_{\delta}\right| \asymp \delta^{d-1}$. For Lemma 2, however, we need

$$
\alpha_{n}=o\left(n^{1 / d-1 / 2} \delta_{n}^{-(d-1) / 2}\right),
$$

in which case we may take $m=K n^{1-1 / d} \alpha_{n}$ for some large $K$. However, (3.8) and (3.9) imply $\alpha_{n}=o\left(n^{(7-3 d) / 8 d}\right)=o(1)$ for $d \geq 3$, so we do not obtain any result at all. (We also need $\alpha_{n} \geq 1$ ). The method of Theorem 2 yields no result either: we obtain $\delta_{n}=K n^{\gamma / 2-1 / 2 d}$ and by (3.9) we have

$$
\alpha_{n}=o\left(n^{(3-d) / 4 d-\gamma(d-1) / 4}\right),
$$

which again contradicts $\alpha_{n} \geq 1$ for any $\gamma>0$ when $d>3$.

We can explain this failure in terms of the heuristics at the beginning of Section 2. We still have a relative variation of the number of points in the strip $S_{\delta}$ of order $n^{-(1-\alpha) / 2}$, for some $\alpha>0$, but this translates to a variation of the height of order only $n^{1 / d-1 / 2+\alpha / 2}$, which does not give any non-trivial result ( $\alpha$ is rather small). Of course, this does not preclude the possibility that there is a substantial variation of the height due to the random position of points in the strip. 


\section{REFERENCES}

1. A. D. Barbour, L. Holst and S. Janson, Poisson Approximation, Oxford Univ. Press, Oxford, 1992.

2. B. Bollobás and G. Brightwell, The height of a random partial order: concentration of measure, Ann. Appl. Probab. 2 (1992), 1009-1018.

3. A. Frieze, On the length of the longest monotone subsequence in a random permutation, Ann. Appl. Probab. 1 (1991), 301-305.

4. J.M. Hammersley, A few seedlings of research, Proc. 6th Berkeley Symp. Math. Stat. Prob. (1972), Univ. of California Press, 345-394.

5. S. Janson, Random coverings in several dimensions, Acta Math. 156 (1986), 83-118.

6. O. Kallenberg, Random Measures, Akademie-Verlag, Berlin, 1983.

7. B.F. Logan and L.A. Shepp, A variational problem for Young tableaux, Advances in Mathematics 26 (1977), 206-222.

8. Michel Talagrand, Concentration of measure and isoperimetric inequalities in product spaces (to appear).

9. S.M. Ulam, Monte Carlo calculations in problems of mathematical physics, Modern Mathematics for the Engineer (1961), E.F. Beckenbach Ed., McGraw Hill, New York.

10. A.M. Veršik and S.V. Kerov, Asymptotics of the Plancherel measure of the symmetric group and the limiting form of Young tableaux, Dokl. Akad. Nauk. SSSR 233 (1977), 1024-1028. (Russian)

Béla Bollobás, Department of Pure Mathematics and Mathematical Statistics, UniverSity of Cambridge, 16 Mill Lane, Cambridge CB2 1SB, England

E-mail address: B.Bollobas@pmms.cam.ac.uk

Svante Janson, Department of Mathematics, Uppsala University, PO Box 480, S-751 06 UPPSAlA, SWEDEN

E-mail address: svante.janson@math .uu.se 